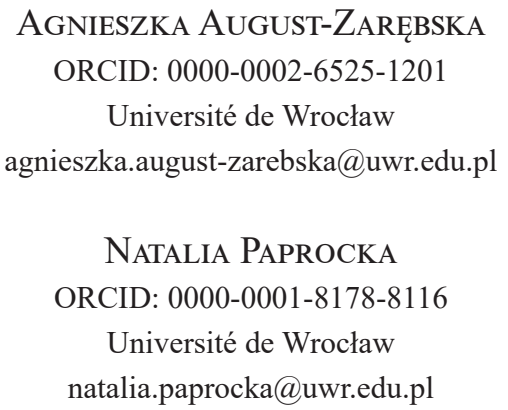

\title{
LES ENJEUX DE LA TRADUCTION LITTÉRAIRE EN LANGUE PÉRIPHÉRIQUE ET POST-VERNACULAIRE. LE CAS DU JUDÉO-ESPAGNOL
}

\section{[...] in translation, Jewish culture is not lost but found ${ }^{1}$.}

\section{INTRODUCTION}

Le judéo-espagnol, dont les racines remontent à la fin du XVI ${ }^{\mathrm{e}}$ siècle, est actuellement classé comme langue en voie de disparition (de 110 à 400000 locuteurs selon les différentes sources), et le développement de la littérature dans cette langue, malgré son histoire cinq fois centenaire, a été brusquement interrompu par l'Holocauste. Cependant, depuis la fin des années 1970, on observe un intérêt croissant pour le maintien de la langue, voire sa revitalisation, aussi bien parmi les Séfarades eux-mêmes que parmi les chercheurs. Cette tendance s'inscrit dans

1 J. Shandler, Adventures in Yiddishland. Postvernacular Language \& Culture, University of California Press, Berkley-Los Angeles-London 2006, p. 92. 
un mouvement plus large visant à conserver et à transmettre l'héritage culturel séfarade $^{2}$. Un des outils les plus importants employés à cette fin est la traduction littéraire.

Dans la présente contribution, nous étudierons les produits de cette dernière pratique, à savoir les traductions contemporaines en langue judéo-espagnole, considérée à la fois comme post-vernaculaire et périphérique (ou dominée), au sens qu'ont donné à ces adjectifs respectivement Jeffrey Shandler ${ }^{3}$, Johann Heilbron $^{4}$ et Pascale Casanova ${ }^{5}$. Cet examen aura pour but de saisir les directions et les spécificités de l'importation littéraire en cette langue, les rôles que jouent ces traductions et les motivations des acteurs engagés dans le processus de traduction.

Le concept de post-vernacularité provient des réflexions de Shandler sur l'état actuel de la culture et de la langue yiddish. Ce chercheur analyse la pratique sociale d'une langue dont le nombre de locuteurs natifs a diminué au point que, pour la plupart des personnes qui l'utilisent encore, elle n'est plus la première langue de communication. Par conséquent, son choix dans certaines situations devient significatif, bien que la hiérarchie sémiotique standard soit inversée : en effet, dans le mode post-vernaculaire, le sens de l'énoncé devient moins important que le fait même qu'il soit formulé dans la langue en question. Ceci résulte du fait que beaucoup de personnes qui l'emploient ne la maitrisent plus parfaitement, voire ne la connaissent plus du tout, mais ressentent pour elle un attachement profond, de la nostalgie, et s'identifient avec son passé6. Ces personnes sont fières d'affirmer que c'était la langue de communication de tous les jours de leurs ancêtres (parfois leurs parents, plus souvent leurs grands-parents ou arrière-grands-parents). Parmi

${ }^{2}$ Nous utilisons le substantif « Séfarade » (et l'adjectif « séfarade») dans le sens plus étroit : pour désigner les Juifs espagnols depuis leur exil et leurs descendants, tandis que leurs ancêtres seront désignés par le terme « Juifs espagnols ». Le sens large et plus familier s'applique également aux Juifs ayant vécu dans la Péninsule ibérique de l'Antiquité jusqu'à l'exil. Au XXe siècle, dans la vie sociopolitique israélienne, on a aussi qualifié de « Juifs séfarades » les Juifs d'Afrique du Nord et d'Asie, malgré leur différence culturelle et historique évidente. Pour plus d'informations sur la terminologie, voir I.M. Hassán, « Los sefardíes: concepto y esbozo histórico », [dans :] P. DíazMas (dir.), Los sefardies. Cultura y literatura, Servicio Editorial Universidad del País Vasco, San Sebastián 1987, pp. 11-22 ; D.L. Gold, « Where have all the Sefardic Jews gone ?», [dans :] P. DíazMas (dir.), op. cit., pp. 143-170 ; S. Della Pergola, «"Sephardic and Oriental" Jews in Israel and Western Countries: Migration, Social Change, and Identification », [dans :] Sephardic Jewry and Mizrahi Jews. Studies in Contemporary Jewry. An Annual XXII, Oxford University Press, OxfordNew York 2007, pp. 3-43.

3 J. Shandler, op. cit.

4 J. Heilbron, «Le système mondial des traductions », trad. de l'anglais par A. Bokobza, [dans :] G. Sapiro (dir), Les contradictions de la globalisation éditoriale, Nouveau monde, Paris 2009, pp. 253-274.

5 P. Casanova, La République mondiale des Lettres, Éditions du Seuil, Paris 1999 ; eadem, "Consécration et accumulation de capital littéraire. La traduction comme échange inégal », Actes de la Recherche en Sciences Sociales 144, 2002, pp. 7-20.

6 J. Shandler, op. cit., pp. 4, 22. 
les pratiques qui peuvent servir à manifester ces émotions, Shandler énumère entre autres la création artistique et la traduction littéraire ${ }^{7}$.

La périphéricité, de même que la centralité qui en est indissociable, sont deux notions utilisées en sociologie de la traduction. Elles sont liées, comme l'explique Heilbron, à la part qu'une littérature donnée occupe dans la macrostructure du marché mondial des traductions littéraires. En effet, «plus un groupe linguistique est central dans le système mondial, plus sa part dans le nombre total de livres traduits dans le monde est grande $\rangle^{8}$. Casanova, elle, préfère évaluer la position d'une littérature donnée dans la «République mondiale des lettres » en termes de domination et de rapports de force. Ainsi, elle oppose des langues dominantes à des langues dominées en fonction du volume de capital linguistico-littéraire qui, lui, dépend du nombre de polyglottes littéraires qui la pratiquent et du nombre de traducteurs littéraires qui font circuler les textes depuis ou vers cette langue littéraire ${ }^{9}$. Parmi les langues dominées, la chercheuse distingue : les langues orales ou dont l'écriture a été récemment fixée (comme certaines langues africaines ou certains créoles) ; les langues de création ou de " recréation » récente, devenues langues nationales (comme le catalan, le coréen, le gaélique, l'hébreu ou le néonorvégien...) ; les langues de culture ou de tradition ancienne liées à de « petits » pays (comme le néerlandais, le danois, le grec ou le persan) ; et finalement les langues de grande diffusion mais peu connues sur le marché littéraire international (comme l'arabe, le chinois ou l'hindi).

Les traductions littéraires en langue judéo-espagnole qui nous intéressent dans le présent article ont déjà fait l'objet d'études ${ }^{10}$, mais jusqu'à présent, les chercheurs se sont intéressés aux traductions plus anciennes, antérieures à la seconde guerre mondiale, c'est-à-dire datant d'une époque où le judéo-espagnol était encore une langue vivante. En revanche, notre étude porte sur les traductions récentes, formulées dans une langue désormais considérée comme en voie de disparition $^{11}$, et publiées sous forme de livre.

7 Ibidem, pp. 24-25, 94.

8 J. Heilbron, op. cit., p. 261.

9 P. Casanova, « Consécration et accumulation de capital littéraire... », p. 9.

10 Voir par exemple R. Singerman, Jewish Translation History. A Bibliography of Bibliographies and Studies, chapitre $8:$ : Judezmo (Ladino) Translations », John Benjamin, Amsterdam-Philadelpia 2002, pp. 323-332 ; J. Linde-Usiekniewicz, M. Kacprzak, Ł. Kołakowski, « Domestykacja i uniwersalizacja w adaptacjach dla młodzieży: polskie i judeohiszpańskie Podróże Guliwera », Prace Filologiczne. Literaturoznawstwo 7(10), 2017, pp. 55-68.

${ }^{11}$ Dans la présente étude, nous analysons uniquement les traductions en judéo-espagnol. Les traductions dans le sens opposé, peu nombreuses, font l'objet de recherches d'Ana Stulić qui les a présentées pendant la journée d'études Éditer et traduire des corpus judéo-espagnols : un enjeu pour la survie de la langue organisée à l'Institut national des langues et civilisations orientales (Inalco) à Paris le 12 novembre 2019. Plus d'informations : <http://www.inalco.fr/evenement/journee-etudesediter-traduire-corpus-judeo-espagnols-enjeu-survie-langue $>$ [consulté le 9.05.2020]. 
Reconstituer le corpus des traductions en une langue menacée de disparition n'est pas facile. Les outils habituellement utilisés pour dresser les bibliographies, tels les catalogues de bibliothèque ou les bases de données (par exemple Index Translationum ${ }^{12}$ ), sont peu efficaces parce que les traductions en question ont été publiées dans différents pays, avec des tirages très faibles et une diffusion très limitée $^{13}$. Les informations qui les concernent passent donc de bouche à oreille entre les chercheurs intéressés, même s'il existe désormais un site, eSefarad, qui s'efforce de les collecter ${ }^{14}$. Il est également difficile d'accéder physiquement à certains livres pour pouvoir les analyser d'autopsie ${ }^{15}$.

Pour pouvoir réaliser nos objectifs, nous procéderons en quatre étapes. (1) Tout d'abord, nous étudierons le contexte historique, c'est-à-dire les racines et l'évolution de la langue judéo-espagnole et de la littérature créée dans cette langue, ainsi que la place qu'ont occupée jadis les traductions par rapport aux œuvres originales sépharades. (2) Ensuite, nous analyserons la situation diamétralement différente de la langue et de la littérature judéo-espagnoles après la seconde guerre mondiale. (3) Ce large contexte esquissé, nous présenterons le corpus établi et nous l'analyserons de trois points de vue : (3.1) celui des œuvres traduites, des langues originales et des éditeurs des traductions, (3.2) celui des traducteurs et de leurs motivations et (3.3) celui des traits caractéristiques des éditions traduites, étant donné que la forme matérielle sous laquelle elles parviennent au destina-

12 La base de données Index Translationum de l'UNESCO ne fait pas de distinction entre le judéo-espagnol et le ladin, langue rhéto-romane parlée dans les Dolomites italiennes, et désigne les deux langues par un seul nom, Ladino. Voir : < http://www.unesco.org/xtrans/> [consulté le 9.05.2020].

13 Les catalogues les plus utiles pour nos recherches ont été ceux des institutions suivantes : la Bibliothèque Nationale d'Israël, la Bibliothèque Nationale de Madrid, la Bibliothèque Tomás Navarro Tomás del Consejo Superior de Investigaciones Científicas, la Bibliothèque du Congrès, la Bibliothèque Nationale de Turquie, le Karlsruhe Virtual Catalog (KVK). Nous avons aussi utilisé la base de données bibliographiques en ligne WorldCat.

14 Créé il y a 10 ans par deux Argentins, Liliana et Marcel Benveniste, le site est disponible à l'adresse <https://esefarad.com/>. Conformément à l'information qui figure sur le site, celui-ci fait partie d'un projet plus vaste qui comprend également un journal électronique, des colloques, un séminaire judéo-espagnol, des émissions à la radio et à la télévision. Voir : <https://esefarad.com/? page_id=5> [consulté le 9.05.2020].

15 Notre corpus n'inclut pas les traductions judéo-espagnoles qui ont pu être réalisées en Turquie dans les années 1950, et quelques rééditions contemporaines de traductions anciennes publiées dans les années 1980 et 1990 aux États-Unis. Cette double exclusion est motivée par les différences d'objectifs et de destinataires de ces deux groupes de traductions par rapport aux traductions de belles-lettres les plus contemporaines visant un public le plus large possible. En revanche, les traductions publiées en Turquie relèvent de la littérature religieuse : elles sont destinées à la liturgie synagogale, à la prière et au développement spirituel des adeptes du judaïsme dans les cercles privés, et les rééditions américaines des traductions des œuvres anciennes, à caractère critique, ont été réalisées par et pour les chercheurs. 
taire détermine directement leur réception ${ }^{16}$. (4) Finalement, nous étudierons les résultats de cette analyse à la lumière croisée des concepts de périphéricité et de post-vernacularité.

\section{CONTEXTE HISTORIQUE}

\subsection{LE JUDÉO-ESPAGNOL EN TANT QUE LANGUE VERNACULAIRE DES JUIFS SÉFARADES}

Le judéo-espagnol, appelé aussi ladino, djudezmo, djidyo, muestro espanyol, était une langue parlée par les Juifs séfarades qui, expulsés d'Espagne à la fin du $\mathrm{XV}^{\mathrm{e}}$ siècle, se sont réfugiés, entre autres, dans les diasporas turco-balkanique et nord-africaine ${ }^{17}$. C'est une langue de fusion créée sur la base de l'espagnol et d'autres langues et dialectes ibéro-romans parlés par les exilés de la Péninsule ibérique ${ }^{18}$. Déjà avant l'exil, leur discours comportait, par rapport à la langue des voisins chrétiens, un fort composant hébreu-araméen qui couvrait principalement les questions religieuses, éthiques ou relatives à l'administration de la communauté. Dans les nouveaux pays d'accueil, le judéo-espagnol s'est transformé sous l'influence des langues locales ${ }^{19}$, en s'enrichissant d'éléments lexicaux et syntaxiques du turc, du grec, du serbo-croate ou du bulgare, ou de l'arabe au Maroc et en Algérie.

${ }^{16}$ Les mêmes corrélations ont été observées par Monika Jaremków par rapport à la réception des traductions polonaises de l'hébreu et de yiddish dans « Polskie przekłady literatury pięknej z języków hebrajskiego i jidysz jako książki (1918-1939) - rekonesans badawczy », Przekładaniec 29, 2014, pp. 118-136.

17 Dans la diaspora occidentale, c'est-à-dire en France, aux Pays-Bas, en Allemagne et en Angleterre, les Séfarades ne parlaient pas un judéo-espagnol vivant. Les deux premières générations après l'exil utilisaient principalement l'espagnol et le portugais comme langues de communication de tous les jours et de culture. Au fil du temps, leurs descendants ont repris les langues nationales dans les deux domaines. Comme dans d'autres centres de la diaspora, l'hébreu et l'araméen dominaient dans la liturgie et dans les études rabbiniques. Cela résultait de la spécificité sociopolitique particulière de cette région. En outre, les communautés de réfugiés de 1492 ont été alimentées par de nouvelles vagues de convertis qui, élevés dans une culture chrétienne hispanophone ou lusophone, ont émigré pour échapper à la persécution de l'Inquisition. Dans leurs nouveaux pays, ils sont revenus au judaïsme et ont intégré les communautés séfarades locales. $C f$. E. Romero, « Historia y literatura », [dans :] I.M. Hassán, R. Izquierdo Benito, E. Romero (dir.), Sefardies: literatura y lengua de una nación dispersa, Ediciones de la Universidad de Castilla-La Mancha, Cuenca 2008, pp. 159 et 165.

18 D.M. Bunis, « Native Designations of Judezmo as a "Jewish Language" », [dans :] Y. Tobi, D. Kurzon (dir.), Hikrei Ma'arav u-Mizrah. Studies in Language, Literature and History Presented to Joseph Chetrit, Carmel Publishing, Jerusalem 2011, p. 41.

19 E. Geller, Jidysz. Język Żydów polskich, Państwowe Wydawnictwo Naukowe, Warszawa 1994, p. 31 
Dans la seconde moitié du XIX ${ }^{\mathrm{e}}$ siècle, les processus d'occidentalisation ${ }^{20}$ se sont intensifiés sur les terres de la première diaspora séfarade. Les changements sont venus, entre autres, des réseaux des écoles européennes - Alliance Israélite Universelle (AIU) et Dante Alighieri - , et des missions chrétiennes. Les communautés séfarades ont alors bénéficié d'un vaste programme d'apprentissage du français et de l'italien, qui ont rapidement acquis un statut de langues de prestige dans les diasporas concernées. Cette période s'est caractérisée par leur influence significative sur la syntaxe et le lexique du judéo-espagnol ${ }^{21}$.

Contrairement à la politique ottomane antérieure, la politique des États-nations qui ont émergé dans la région turco-balkanique était axée sur l'unification ethnique, linguistique et culturelle de la société. En résultat, dès la fin du XIX ${ }^{\mathrm{e}}$ siècle, la langue vernaculaire des Séfarades a peu à peu cédé la place aux langues véhiculaires de prestige (français, italien, espagnol), puis aux langues des pays concernés (turc, grec, serbo-croate, bulgare...). L'emploi du judéo-espagnol est ainsi devenu de plus en plus restreint au milieu familial. Cette tendance s'est maintenue dans les pays de la diaspora secondaire où les Juifs séfarades émigraient pour des raisons économiques ou idéologiques. Dans les Balkans, le nombre d'utilisateurs du judéo-espagnol a considérablement diminué durant la seconde guerre mondiale, quand la communauté juive parlant cette langue a été presque entièrement anéantie lors de la Shoah.

\subsection{ANCIENNE LITTÉRATURE JUDÉO-ESPAGNOLE : À LA PÉRIPHÉRIE DU SYSTÈME MONDIAL DES LETTRES}

Le statut particulier de l'ancienne littérature judéo-espagnole provient de son triple enracinement dans la littérature d'Espagne, dans la littérature des terres d'accueil et leurs traditions multiples, et enfin, dans la littérature d'Israël, avec son héritage civilisationnel et sa langue. Les premiers monuments littéraires formulés dans cette langue, très peu nombreux, proviennent encore de la péninsule ibérique et datent des XIVe et $\mathrm{XV}^{\mathrm{e}}$ siècles $^{22}$; on dispose aussi d'un petit nombre de textes $\mathrm{du} \mathrm{XVI}^{\mathrm{e}}$ siècle, imprimés principalement à Constantinople et à Thessalonique, ainsi que des responsa rabbiniques et des traductions de la littérature éthique et morale (musar) de l'hébreu du XVII ${ }^{\mathrm{e}}$ siècle.

20 À cette époque, il s'est produit une transition de la communauté traditionnelle (où la majorité des sphères de la vie étaient réglementées par la religion, avec une structure administrative résultant de la religion et où les élites administratives étaient également religieuses) à une société moderne, sécularisée, de plus en plus intégrée à la société du pays d'accueil, ou partageant la vision du monde laïque du sionisme.

${ }^{21}$ D.M. Bunis, "The Language of the Sephardim. A Historical Overview », [dans :] H. Beinart (dir.), Moreshet Sepharad: the Sephardi Legacy, t. 2, The Magnes Press, Jerusalem 1992, p. 411 ; E. Romero, op. cit., pp. 178-179.

22 D.M. Bunis, « The Language of the Sephardim... », p. 402. 
Pendant la majeure partie de son histoire, la littérature séfarade a été, comme le constate Olga Borovaia, imitative ou ré-écrite (re-written), parce qu'elle se basait principalement sur des traductions et des adaptations ${ }^{23}$. De ce fait, dans le polysystème de la littérature judéo-espagnole, les traductions ont toujours occupé la position centrale ${ }^{24}$. Cependant, les directions de l'importation littéraire ont varié au cours des siècles. Dans la phase de développement initiale, on traduisait principalement de l'hébreu, moins souvent de l'araméen, ce qui était étroitement lié à la diglossie propre aux cultures juives, dans lesquelles l'hébreu et l'araméen étaient les langues de la liturgie, de la religion et de la pensée éthique, tandis que les autres langues étaient utilisées pour la communication quotidienne. Au XVIII ${ }^{\mathrm{e}}$ siècle, qui est considéré comme le temps de la consolidation du judéo-espagnol en tant que langue d'expression artistique et le temps de l'essor de la littérature dans cette langue ${ }^{25}$, les traductions, toujours de l'hébreu, des textes religieux, mystiques, moraux, philosophiques et historiques continuent à jouer un rôle important. Ce n'est que dans la seconde moitié du XIX ${ }^{\mathrm{e}}$ et au début du $\mathrm{XX}^{\mathrm{e}}$ siècles que les centres d'importation littéraire changent, avec le développement de la littérature laïque, largement déterminé par le rôle croissant de la presse. C'est surtout dans les magazines que sont publiées les traductions — d'habitude abrégées, compilées ou adaptées - des œuvres de la littérature européenne, avant tout française : récits, romans et autres textes en prose, pièces de théâtre et poèmes ${ }^{26}$.

Contrairement à cette importation littéraire florissante, l'exportation de la littérature séfarade a toujours été proche de zéro ${ }^{27}$, ce qui l'a cantonnée dans la périphérie du système mondial de traduction. Le capital linguistico-littéraire de cette littérature dominée a toujours été faible, si on le mesure, comme le veut Casanova, au volume de prestige spécifique, à l'ancienneté de la langue et au nombre de textes déclarés universels écrits dans cette langue ${ }^{28}$.

${ }^{23}$ O. Borovaia, «The Role of Translation in Shaping the Ladino Novel at the Time of Westernization in the Ottoman Empire (A Case Study: Hasan-Pasha and Pavlo y Virzhinia) », Jewish History 16(3), 2002, pp. 263-264.

${ }^{24}$ Nous nous référons au concept de polysystème développé par I. Even-Zohar, «The Position of Translated Literature within the Literary Polysystem », [dans :] J. Holmes, J. Lambert, R. van den Broeck (dir.), Literature and Translation. New Perspectives in Literary Studies, Acco, Leuven 1978, pp. 117-127.

25 E. Romero, op. cit., pp. 170-177 ; eadem, « Sephardi coplas. Characteristics and Bibliography », European Judaism 44(1), 2011, p. 72.

${ }^{26}$ D.M. Bunis, « The Language of the Sephardim... », p. 411.

27 O. Borovaia, op. cit., p. 265.

${ }^{28}$ P. Casanova, "Consécration et accumulation de capital littéraire... », p. 9. 


\section{CONTEXTE ACTUEL : LANGUE POST-VERNACULAIRE ET LITTÉRATURE EN DEHORS DES PÉRIPHÉRIES ?}

Après la seconde guerre mondiale, le statut du judéo-espagnol et de la littérature produite dans cette langue a radicalement changé. Dans les pays des Balkans, les Juifs qui n'avaient pas émigré ont cessé de la parler à leurs enfants, car elle était trop associée à la langue qui avait permis d'identifier les Juifs pendant 1'Holocauste. Elle servait donc d'outil de communication entre gens de la génération précédente et de langage " secret » utilisé par les adultes pour discuter de sujets que les enfants n'étaient pas censés entendre. En outre, dans les pays de la diaspora primaire comme de la secondaire, la connaissance des langues nationales était déterminante pour trouver du travail, accéder à l'éducation, et surtout, gagner une bonne position sociale. C'est ainsi que pendant les décennies suivantes, la presse et la littérature judéo-espagnoles, jadis si florissantes, ont presque complètement cessé d'exister. On ne traduisait plus ni en judéo-espagnol, ni de cette langue.

Le changement n'est venu qu'à la fin des années 1970, lorsqu'il est devenu évident que la langue et la littérature judéo-espagnoles étaient en déclin. Les chercheurs prédisaient que leur fin viendrait avec la mort de la génération des anciens $^{29}$. Un certain nombre de mesures ont alors été prises pour documenter la langue avant qu'elle ne disparaisse complètement. En même temps, les locuteurs natifs et leurs descendants, qui souvent n'avaient déjà plus toutes les compétences linguistiques, ont entrepris les premières tentatives d'écriture littéraire (principalement poétique) après une longue période de silence. La période qui suit la fin des années 1970 se caractérise par un regain d'intérêt pour la langue et l'ethnie séfarades connu sous le nom d'arrebivimiento. Cette tendance, avec des variations, s'est poursuivie jusqu'à nos jours. Les auteurs contemporains qui ont choisi le judéo-espagnol comme langue d'expression artistique publient principalement dans la revue Aki Yerushalayim, fondée en 1979, et dans d'autres magazines multilingues à thème séfarade. Certains ont publié des recueils de poésie mono ou bilingues où le judéo-espagnol coexiste avec l'hébreu, l'anglais ou l'espagnol. Mais vers la fin du $\mathrm{XX}^{\mathrm{e}}$ siècle, cette production originale a commencé à s'arrêter, « remplacée » au début du XXI ${ }^{\mathrm{e}}$ siècle par des traductions littéraires en judéo-espagnol.

\section{LES TRADUCTIONS CONTEMPORAINES EN JUDÉO-ESPAGNOL}

\subsection{CEUVRES, LANGUES, ÉDITEURS, PAYS}

Seules sept œuvres étrangères différentes ont été traduites en judéo-espagnol à l'époque contemporaine, dont une a été publiée deux fois, une autre, en deux vo-

29 H.V. Sephiha, L'agonie des Judéo-espagnols, Éditions Entente, Paris 1991 [1977], pp. 107-108. 
lumes, et deux autres encore, dans deux versions linguistiques ou alphabétiques ${ }^{30}$. On a aussi traduit deux anthologies et un recueil de poésie. La première traduction moderne en judéo-espagnol est parue en 2006, et jusqu'à la fin de 2019, quinze volumes de traductions ont été publiés. Les œuvres traduites étaient à l'origine écrites en hébreu, grec (ancien et moderne), yiddish, français, anglais et espagnol. La plupart des traductions judéo-espagnoles ont été publiées par des éditeurs de niche installés dans six différents pays. La plupart d'entre eux (quatre) organisent leur activité autour de la promotion de la culture judéo-espagnole. Cet objectif est commun à trois maisons d'édition israéliennes (Yeriot, Sentro Moshe David Gaon de Kultura Djudeo-Espanyola et Ha-reshut ha-leumit le-tarbut ha-ladino/Autoridad Nasionala del Ladino i su Cultura) et une argentine (Centro de Investigación y Difusión de la Cultura Sefardí). Les deux autres s'intéressent à la diversité linguistique et publient des livres en langues minoritaires (Evertype ${ }^{31}$, en Irlande, et Tintenfass ${ }^{32}$, en Allemagne). Il existe aussi un site indien, Cyberwit.net, qui a pour vocation de " publier d'excellentes œuvres de poésie qui ne trouveront probablement pas leur place ailleurs $»^{33}$. Le seul éditeur généraliste à avoir publié des traductions judéo-espagnoles est la maison d'édition espagnole Herder.

Le corpus établi (voir : Annexe) peut sembler extrêmement modeste pour une langue de tradition séculaire, mais le rythme de publication des traductions s'est considérablement accéléré depuis 2006, notamment dans la deuxième décennie du XXI siècle. La parution presque parallèle de quinze volumes peut être interprétée principalement comme une continuation du phénomène d'arrebivimiento, mais aussi comme le résultat de l'activité des chercheurs et des activistes, conscients de plus en plus du rôle que la traduction joue dans le processus de sauvegarde d'une langue en danger.

\subsubsection{LITTÉRATURE DE LA SHOAH (4 VOLUMES)}

Compte tenu de l'histoire des Juifs séfarades, les premiers choix traductifs ne surprennent pas : ils relèvent de la littérature de la Shoah. En 2006, paraît la traduction du Chant du peuple juif assassiné d'Ytshak Katzenelson, poème narratif considéré comme l'un des témoignages littéraires les plus remarquables de l'Holocauste et un chef-d'œuvre de la littérature mondiale. Construit en quinze chants, il a été écrit entre 1943 et 1944 au camp de Vittel, en France, après trois ans de lutte dans le ghetto de Varsovie et avant la déportation de l'auteur à Auschwitz. La traduction en judéo-espagnol est parue d'abord dans une édition trilingue

${ }^{30}$ Les données bibliographiques complètes des traductions en judéo-espagnol se trouvent à la fin de l'article.

31 Voir le site : <https://www.evertype.com/> [consulté le 22.10.2020].

32 Voir le site : <https://www.verlag-tintenfass.de/index.html $>$ [consulté le 22.10.2020].

33 Voir le site : <https://www.cyberwit.net/about.htm> [consulté le 22.10.2020]. 
(avec l'original en yiddish et la traduction espagnole), et deux ans plus tard (2008), en version unilingue.

Le sujet de la Shoah est également abordé dans deux volumes écrits par des auteurs rescapés de l'Holocauste et des camps de la mort : Las Angustias del Enferno (2007) de Moshe 'Ha-Elion (né en 1925) et Yo esto reklamando! (2014) de Moshe Liba (né en 1931).

\subsubsection{LITTÉRATURE DE JEUNESSE (6 VOLUMES)}

La littérature de jeunesse parait beaucoup moins évidente comme choix traductif, compte tenu de l'absence de destinataires naturels des traductions judéo-espagnoles. En effet, il n'y a plus d'enfants pour qui cette langue constitue la première langue de socialisation ou à qui on l'aurait enseignée parmi plusieurs autres langues.

Deux des œuvres de cette catégorie sont des classiques pour la jeunesse. La traduction judéo-espagnole du Petit Prince d'Antoine de Saint-Exupéry est la seule du corpus analysé à avoir été rééditée deux fois, en 2010 et 2012. Elle contient le texte judéo-espagnol en deux versions alphabétiques : en écriture Rachi et en transcription latine. Alice au pays des merveilles existe, elle aussi, en deux versions alphabétiques, mais chacune d'entre elles a été publiée séparément, à un intervalle de deux ans (en 2014 et 2016). Les deux traductions judéo-espagnoles faisaient partie de projets internationaux visant à publier les œuvres en question dans le plus grand nombre de langues possible.

Deux anthologies de la poésie hébraïque intitulées Yeladino et Yeladino 2 (2017, 2018) s'adressent également aux enfants. Ces publications font partie d'un projet plus vaste qui inclut également un spectacle et un profil Facebook ${ }^{34}$. Les volumes sont enrichis d'illustrations modernes et d'un $\mathrm{CD}$ avec des enregistrements de poèmes sous forme de chansons. Les volumes sont bilingues, combinant la version originale en hébreu avec une traduction judéo-espagnole en écriture carrée.

\subsection{3. ÉPOPÉES (4 VOLUMES)}

Viennent ensuite trois épopées d'envergure nationale. Traduites du grec ancien, l'Odyssée (en deux volumes) et l'Iliade (un volume pour l'instant) d'Homère ont été publiées en édition bilingue hébreu-judéo-espagnole. Cette traduction a permis d'introduire dans les deux polysystèmes littéraires deux œuvres canoniques de la littérature européenne. Le projet a été motivé par le besoin d'élargir

${ }^{34}$ Voir le site : <https://www.facebook.com/yeladinoANL/> [consulté le 9.05.2020]. 
les moyens linguistiques et artistiques du judéo-espagnol, ce que la traduction des vastes poèmes épiques a permis de réaliser ${ }^{35}$. La traduction de textes d'un tel prestige en judéo-espagnol a également servi de moyen de consécration de cette langue post-vernaculaire qui, à la fin du XIX ${ }^{\mathrm{e}}$ siècle, n'était parfois considérée que comme un jargon.

La troisième épopée, El gaucho Martín Fierro de José Hernández, est considérée comme l'exemple même du style gaucho et le livre national des Argentins. La figure du gaucho incarne la lutte contre l'injustice sociale résultant des circonstances historiques du pays. Elle a été élevée au rang de héros national, représentante de l'argentinité mythique (argentinidad), dotée des caractéristiques et des valeurs appréciées par une société qui a réussi à se libérer de la domination coloniale espagnole. Le choix de cette œuvre par le traducteur Carlos Levy constitue un acte de reconnaissance d'identité complexe : il se sent pleinement Argentin, mais en même temps reconnaît ses racines séfarades ${ }^{36}$.

\subsubsection{POÉSIE (1 VOLUME)}

Le corpus de traductions englobe également un petit recueil de poésie hébraïque contemporaine Abondansa del miel detenido: seleksion de poemas 19482008 d'El'azar Granot, traduits par Avner Perez. Sa publication en judéo-espagnol témoigne de la conception qu'a le traducteur du rôle de la traduction dans la phase actuelle de la langue et de la littérature séfarades. Selon lui, le travail sur la traduction élargit les moyens linguistiques et artistiques d'une langue, et d'autre part, l'augmentation du nombre de textes disponibles permet aux lecteurs d'entretenir leurs compétences linguistiques ${ }^{37}$.

\subsection{TRADUCTEURS}

Les noms des sept traducteurs judéo-espagnols figurent dans les volumes concernés. Il s'agit d'Avner Perez (6 volumes), Moshe 'Ha-Elion (4 volumes), Shmuel Refael, Gladys Pimienta et Arnau Pons (2 volumes chacun), ainsi que de Zelda Ovadia et Carlos Levy (un volume chacun). Les traducteurs qui ont ladinisé

35 A. Perez, « La fuersa de la traduksion. Sovre las relasiones mutuales entre traduksion i ovra orijinal en ladino ", [dans :] Homère, La Odisea (Kantes XIII-XXIV), Yeriot, Ma'ale Adumim 2014, pp. VII-VIII.

36 C. Levy, [Préface sans titre], [dans :] J. Hernández, El Gaucho Martín Fierro, Centro de Investigación y Difusión de la Cultura Sefardí, Buenos Aires 2017, p. 7.

37 A. Perez, « Sovre la traduksion de ovras klasikas en ladino i su influensia sovre la kreasion orijinal », Aki Yerushalayim 96, 2014, <http://www.akiyerushalayim.com/ay/096/096_12_sovre. htm> [consulté le 9.05.2020]. 
la littérature étrangère sont pour la plupart des Séfarades qui ressentent un fort attachement pour leur langue et leur culture et qui perçoivent leur activité de traduction comme un des moyens de les préserver. Ils ont commencé à traduire en judéo-espagnol généralement après avoir atteint un âge mûr, voire avancé (comme Moshe 'Ha-Elion qui a traduit les deux épopées d'Homère à l'âge de 90 ans !). Il y a parmi eux des rescapés de l'Holocauste (témoins directs) et des descendants de rescapés (deuxième génération), des chercheurs dans le domaine de la culture séfarade et des militants soucieux de la préserver.

Les traducteurs les plus productifs, Avner Perez (né en 1942 en Israël) et Moshe 'Ha-Elion (né en 1925 à Thessalonique), sont aussi les plus emblématiques de ce groupe. L'activité prolifique et polyvalente du premier vise entièrement à la revitalisation de la langue et de la culture judéo-espagnole. À part la traduction, il est chercheur en culture séfarade ${ }^{38}$ et coauteur de l'un des rares dictionnaires judéo-espagnols contemporains ${ }^{39}$. En 1992, il a fondé l'Institut Ma'ale Adumim, qui a pour vocation de documenter la culture judéo-espagnole. Il est également connu de son œuvre poétique en judéo-espagnol. En tant que traducteur de cette langue, il a traduit Le Petit Prince, Alice au pays des merveilles et les poèmes réunis dans les volumes Abondansa del miel detenido (2008) d'El'azar Granot. Sa maison d'édition, Yeriot, a publié plusieurs œuvres séfarades, y compris les traductions judéo-espagnoles de l'Iliade et de l'Odyssée par 'Ha-Elion. Perez tenait particulièrement à ce traducteur, parce que celui-ci est natif du judéo-espagnol et du grec, et connaît bien aussi le grec ancien. Survivant des camps de concentration d'Auschwitz, Mauthausen, Melk et Ebensee, 'Ha-Elion a choisi le judéo-espagnol comme langue d'expression artistique de ses propres œuvres ${ }^{40}$. Il est aussi l'auteur et en même temps l'auto-traducteur en judéo-espagnol de ses souvenirs des camps, Las Angustias del Enferno (2007) (publiés originellement en grec).

\subsection{TRAITS CARACTÉRISTIQUES DES TRADUCTIONS JUDÉO-ESPAGNOLES}

La réception des traductions contemporaines en judéo-espagnol publiées sous forme de livre se distingue par trois traits caractéristiques : la diversité typographique, la diversité linguistique et l'abondance des paratextes.

38 Pour son activité de recherche, Perez a reçu le prix Samuel Toledano en 2008, une haute distinction dans le domaine de la recherche sur l'histoire et la culture des Séfarades et leurs relations avec l'Espagne (<http://folkmasa.org/av/toledanoc.htm> [consulté le 9.05.2020]).

39 A. Perez, G. Pimienta, Diksionario Amplio Djudeo-espanyol - Ebreo. Lashon me-Aspamia, Sefarad - El Instituto Ma'ale Adumim, La Autoridad Nasionala del Ladino i su Kultura, Ma'ale Adumim 2007. Version élargie du dictionnaire est accessible en ligne : <http://folkmasa.org/ milon/pmilonl.htm> [consulté le 9.05.2020].

40 M. 'Ha-Elion, En los Kampos de la Muerte, Instituto Ma'ale Adumim, Ma'ale Adumim 2000 ; idem, Tres mujeres en el kamino de pedrisión-Saloniki-Birkenau: drama, [lieu et maison d'édition inconnus] 2016. 


\subsubsection{DIVERSITÉ TYPOGRAPHIQUE}

La grande diversité typographique de traductions contemporaines judéoespagnoles résulte du fait qu'actuellement, cette langue utilise deux alphabets différents, le latin et l'hébreu, ce dernier présentant en plus deux variétés d'écriture : la Rachi, utilisée en imprimerie principalement du XVIII ${ }^{\mathrm{e}}$ jusqu'à la première moitié du $\mathrm{XX}^{\mathrm{e}}$ siècle, et la carrée, propre à l'hébreu moderne et utilisée en judéo-espagnol surtout dans les titres ${ }^{41}$.

Le choix de l'alphabet entraîne d'autres divergences, car le sens d'écriture et de lecture des alphabets latin et hébreu est opposé (respectivement dextro et sinistroverse). Les livres s'ouvrent donc de l'autre côté : la tradition éditoriale européenne veut que le dos du livre se trouve à gauche, et la tradition hébraïque, qu'il se trouve à droite. Quand un même volume contient deux versions alphabétiques du texte judéo-espagnol, le livre peut être lu des deux côtés et possède donc deux premières de couverture, deux pages de garde, deux pages de titre, etc. C'est le cas, par exemple, du Petit Prince judéo-espagnol : d'un côté du livre, le texte est en alphabet latin et de l'autre, en écriture Rachi, les deux parties ayant une pagination indépendante.

La division du livre entre deux écritures, et donc deux sens de lecture, n'est pas toujours symétrique (moitié-moitié). Parfois, dans un livre répondant à l'une des traditions éditoriales, une partie des pages seulement se lisent dans le sens opposé. C'est par exemple le cas pour l'anthologie Yeladino 2, qui suit la tradition éditoriale hébraïque. Les poèmes traduits, en écriture carrée, doivent être lus de droite à gauche et le dos du livre est donc à droite pendant la lecture. Cependant, une vingtaine de pages (45-65) contiennent les mêmes traductions, mais en écriture latine, elles se lisent donc dans le sens opposé. Le sens de lecture des pages ne correspond d'ailleurs pas forcément à celui de l'alphabet utilisé. Par exemple, la préface à la traduction judéo-espagnole d'Alice au pays des merveilles doit être lue selon la tradition hébraïque, bien qu'elle soit écrite en anglais !

\subsubsection{COEXISTENCE DU JUDÉO-ESPAGNOL AVEC D’AUTRES LANGUES}

La caractéristique suivante des traductions analysées est la coexistence fréquente du judéo-espagnol avec d'autres langues, ce qui s'applique aussi bien au

41 L'alphabet latin, moins utilisé au début, s'est répandu en Bosnie et en Serbie au début du $\mathrm{XX}^{\mathrm{e}}$ siècle et en Turquie après 1928, soit après la réforme de Kemal Atatürk qui réglementait l'utilisation d'un seul alphabet pour tous les groupes ethniques de la jeune république. La cursive Rachi a été plus longtemps utilisée à Thessalonique, où de nombreuses imprimeries séfarades ont fonctionné jusqu'à la seconde guerre mondiale. Malheureusement, ces institutions ne se sont pas rétablies après la Shoah. Depuis lors, l'alphabet latin domine dans les rares périodiques et livres et, au cours des dernières décennies, sur les sites Web. La situation se complique encore par l'utilisation de différents systèmes orthographiques. Le judéo-espagnol n'a connu aucune codification officielle, ni avant ni après la seconde guerre mondiale. Voir : D.M. Bunis, « The Language of the Sephardim... », pp. 400-401. 
texte principal qu'aux paratextes qui l'entourent. Ainsi, la traduction judéo-espagnole du Chant du peuple juif assassiné de Katzenelson, précédée de la version yiddish originale, s'accompagne en plus de la traduction espagnole. Les deux volumes de Yeladino sont, eux, bilingues : les poèmes originaux en hébreu sont suivis de leurs traductions judéo-espagnoles.

Le texte principal en judéo-espagnol coexiste souvent avec des paratextes en d'autres langues. Par exemple, dans les deux éditions judéo-espagnoles d'Alice au pays des merveilles, le prologue du traducteur Avner Perez figure en deux versions linguistiques, anglaise et judéo-espagnole, qui, elle, est en outre en deux versions alphabétiques.

Des langues autres que le judéo-espagnol, principalement l'anglais et l'espagnol, sont souvent aussi présentes sur les pages de titre et de garde (pour fournir des informations telles que le nom de l'auteur et du traducteur, le titre de l'original, le lieu de publication, les mentions légales, l'ISBN, etc.). En général, il s'agit des langues utilisées par les maisons d'édition et de contact avec les clients. Les informations dans ces langues, à caractère bibliologique et commercial, facilitent l'accès au livre aux personnes qui ne connaissent pas le judéo-espagnol et le classement dans les bibliographies et catalogues.

\subsubsection{ABONDANCE PARATEXTUELLE}

Les traductions judéo-espagnoles s'accompagnent d'habitude de nombreux paratextes qui proviennent des auteurs ou de personnages liés à la politique, à la culture ou aux recherches ${ }^{42}$. Souvent, dans un même volume, plusieurs paratextes se suivent : par exemple, le livre Yo esto reklamando!, outre la traduction de 56 poèmes de Moshe Liba, contient aussi une préface de l'auteur, traduite de l'hébreu et complétée d'informations sur la traduction judéo-espagnole ${ }^{43}$, deux préfaces de personnages politiques, Shimon Peres ${ }^{44}$ et Yitzhak Navon ${ }^{45}$, et une postface d'Ada Aharoni de 1'Université de Haïfa ${ }^{46}$.

${ }^{42}$ Par exemple, l'édition trilingue du Chant du peuple juif assassiné contient l'épilogue de Philippe Mesnard, affilié à l'Université de Clermont-Ferrand, spécialiste de la littérature de la Shoah («Reconocer la voz de ese grito », [dans :] I. Katzenelson, Dos lid funem oisgueharguetn ídishn folk. El canto del pueblo judio asesinado. El kante del puevlo dijdyó atemado, Herder, Barcelona 2006, pp. 259-269).

${ }^{43}$ M. Liba, «Introduksion al livro Ani Tovea! - Yo esto reklamando! en ladino », [dans :] idem, Yo esto reklamando!, Cyberwit.net, Allahabad 2014, pp. 11-13.

44 S. Peres, « Prefasio al livro: De "Bet'Ha'am" al "Binyanei `Hauma", poezias, Moshé Liba, 1987. Moshe Liba — poeta del dolor i de la esperansa », [dans :] M. Liba, Yo esto reklamando!, p. 3.

45 Y. Navon, "Prefasio al livro: El violinista de Auschwitz, poezia, Moshe Liba, 1987. Strumza i Liba - El violinista de Auschwitz i el poeta de Yerushalayim », [dans :] M. Liba, Yo esto reklamando!, pp. 4-5.

46 A. Aharoni, «La poezia emosionante de Moshé Liba », [dans :] M. Liba, Yo esto reklamando!, pp. 6-10. 
Enfin, les traducteurs judéo-espagnols s'expriment eux-mêmes volontiers dans des paratextes divers. C'est le cas dans onze des quinze éditions de traductions judéo-espagnoles relevées. Les traducteurs y mentionnent souvent des autorités dans le domaine de la langue judéo-espagnole avec qui ils ont coopéré, mais avant tout, ils dévoilent les raisons qui les ont poussés à traduire les œuvres étrangères concernées. Trois types de motivations se laissent dégager. Tout d'abord, conscients du passé tragique des Séfarades, les traducteurs veulent, à l'aide de la traduction, commémorer la langue et la culture des ancêtres ou donner un témoignage de l'Holocauste. Ensuite, leurs motivations sont parfois liées à leur état d'esprit actuel et à leur identité complexe, qu'ils veulent manifester par la traduction. Et finalement, certaines de leurs motivations s'orientent vers l'avenir, car elles manifestent un désir de prolonger l'existence de la langue et de la littérature séfarade en voie de disparition.

\section{CONCLUSIONS}

Considérer la traduction littéraire en langue judéo-espagnole à partir de son volume de capital linguistico-littéraire est vain, puisque celui-ci n'existe quasiment pas. Remarquons que le modèle de Casanova ne prévoit même pas de place pour des langues telles que le judéo-espagnol : avec une longue histoire, mais sans pays ni diffusion. C'est une langue qui est entrée dans sa phase de déclin et qui, pour un certain temps, a cessé de participer à l'échange littéraire transnational, disparaissant même de la périphérie du système mondial de traduction. Cependant, elle n'est pas complètement à l'agonie, elle est entrée dans une phase de développement qui peut être décrite, d'après Shandler, comme post-vernaculaire. L'objectif majeur des acteurs engagés dans la traduction vers une langue arrivée à ce stade est de la faire survivre.

Le statut post-vernaculaire du judéo-espagnol, caractérisé par un fort attachement à une langue souvent mal ou pas du tout connue, détermine également la forme et le fonctionnement des traductions en judéo-espagnol publiées sous forme de livre. Dans les volumes analysés, les textes des traductions sont imprimés en différents alphabets et, dans le cas de l'alphabet hébreu, en différentes écritures, car le judéo-espagnol ne dispose pas même d'un système d'écriture universellement accepté. Cette tendance éditoriale peut résulter de la volonté d'atteindre plus de lecteurs. Les traductions coexistent presque toujours avec des textes dans d'autres langues : avec des originaux, d'autres traductions, et de nombreux paratextes dont le rôle est d'introduire et de commenter, voire d'expliquer les traductions judéo-espagnoles. Cela donne l'impression que, du fait du mauvais état de la langue, ces traductions seraient inaptes à fonctionner de manière indépendante et exigeraient toujours un support sous forme de paratexte ou d'une autre version linguistique plus explicite. Une telle manière de publier les traductions judéo-espagnoles indique qu'elles sont destinées également à des personnes qui 
ne connaissent pas bien la langue, mais souhaitent avoir des objets liés à la culture séfarade. Pour elles, le livre contenant du texte en judéo-espagnol est un objet d'affection qui leur permet d'entretenir et de manifester le souvenir de leur origine et leur relation avec une culture séculaire, y compris sur le plan linguistique.

La nature périphérique de la langue et de la littérature judéo-espagnoles d'aujourd'hui se manifeste à son tour par une absence quasi-totale de directions d'emprunt privilégiées (centres), ce qui peut être lié à la minceur du corpus actuel. La production de traductions judéo-espagnoles se caractérise également par une grande dispersion géographique : en effet, les traductions sont publiées dans des lieux éloignés, ce qui résulte du passé diasporique de la branche culturelle séfarade, mais aussi de la nature post-vernaculaire de la langue judéo-espagnole et de son absence de centre culturel commun.

Tout comme dans d'autres littératures juives ${ }^{47}$, dans le polysystème de la littérature séfarade ancienne, les traductions ont toujours occupé une position centrale, seuls les langues et genres préférés des textes traduits changeaient. En même temps, cette littérature s'est toujours située à la périphérie du système mondial de traduction, car sa contribution aux échanges littéraires transnationaux était minime.

Aujourd'hui aussi, les traductions semblent jouer un rôle important dans la littérature judéo-espagnole contemporaine, bien que le corpus de textes traduits soit (encore) petit et que ni langue ni genre préférés ne puissent être distingués. Cependant, les traductions constituent aujourd'hui l'un des outils les plus importants pour maintenir en vie cette langue en voie de disparition et sa littérature en déclin. C'est particulièrement vrai à un moment où cette littérature ne connaît pratiquement plus d'apport d'œuvres originales. On ne sait pas comment la situation va évoluer à l'avenir. Il est probable que de rares traductions, peut-être accompagnées de quelques œuvres originales, continueront d'être produites jusqu'à l'extinction finale de la langue. Cependant, il n'est pas non plus exclu que les traductions enrichissent et « raniment » cette langue affaiblie et contribuent à faire renaître sa littérature.

\section{ANNEXE}

Liste des traductions contemporaines en langue judéo-espagnole ${ }^{48}$

Carroll L., Las Aventuras de Alisia en el Paiz de las Maraviyas, trad. en judéo-espagnol A. Perez, Evertype, Leac an Anfa-Cathair na Mart 2014.

47 Cf. Z. Shavit, « The Status of Translated Literature in the Creation of Hebrew Literature in Pre-State Israel (The Yishuv Period) », Meta 43(1), 1997, pp. 1-8; G. Toury, « Hebrew Tradition », [dans :] M. Baker, K. Malmkjær (dir.), Routledge Encyclopedia of Translation Studies, Routledge, London 1998, pp. 439-448.

48 Les noms d'auteurs et de lieux d'édition sont donnés en version française si elle existe. Les titres et autres éléments bibliographiques en alphabet hébreu, aussi bien en écriture carrée qu'en Rachi, sont transcrits en alphabet latin conformément à la version indiquée sur les couvertures. 
Carroll L., Las Aventuras de Alisia en el Paiz de las Maraviyas, trad. en judéo-espagnol A. Perez, Evertype, Portlaoise 2016.

Granot E., Abondansa del miel detenido: seleksion de poemas 1948-2008, trad. en judéo-espagnol A. Perez, Yeriot, Ma'ale Adumim 2007.

'Ha-Elion M., Las Angustias del Enferno: las pasadias de un djidio de Saloniki en los kampos de eksterminacion almanes Auschwitz, Mauthausen, Melk, Ebensee, trad. en judéo-espagnol M. 'Ha-Elion, Sentro Moshe David Gaon de Kultura Djudeo-Espanyola, Universidad BenGurion del Negev, Beer-Sheva 2007.

Hernández J., El Gaucho Martín Fierro, trad. en judéo-espagnol C. Levy, Centro de Investigación y Difusión de la Cultura Sefardí, Buenos Aires 2017.

Homère, La Iliada (Kantes I-XII), trad. en judéo-espagnol M. 'Ha-Elion, trad. en hébreu A. Perez, Yeriot, Ma'ale Adumim 2016.

Homère, La Odisea (Kantes I-XII), trad. en judéo-espagnol M. 'Ha-Elion, trad. en hébreu A. Perez, Yeriot, Ma'ale Adumim 2011.

Homère, La Odisea (Kantes XIII-XXIV), trad. en judéo-espagnol M. 'Ha-Elion, trad. en hébreu A. Perez, Yeriot, Ma'ale Adumim 2014.

Katzenelson I., Dos lid funem oisgueharguetn idishn folk. El canto del del pueblo judio asesinado. El kante del puevlo dijdyó atemado, trad. en castellan et transcription en yiddish E. Toker, trad. en judéo-espagnol A. Pons, Herder, Barcelona 2006.

Katzenelson I., El kante del puevlo dijdyó atemado, trad. en judéo-espagnol A. Pons, Herder, Barcelona 2008.

Liba M., Yo esto reklamando!, trad. en judéo-espagnol Z. Ovadia, Cyberwit.net, Allahabad 2014.

Saint-Exupéry A. de, El Princhipiko, trad. en judéo-espagnol A. Perez, G. Pimienta, Edition Tintenfass, Neckarsteinach 2010.

Saint-Exupéry A. de, El Princhipiko, trad. en judéo-espagnol A. Perez, G. Pimienta, Edition Tintenfass, Neckarsteinach 2012 [éd. 2].

Yeladino: Shirei yaldut israelit be-targum le-ladino. Kantigas ebreas para ninyos traduzidas $i$ kantadas en ladino. Cantigas hebreas para niños traducidas y cantadas en Ladino, trad. en judéo-espagnol S. Refael, Ha-reshut ha-leumit le-tarbut ha-ladino/Autoridad Nasionala del Ladino i su Cultura, Jérusalem 2017.

Yeladino 2: Od shirei yaldut israelit be-targum le-ladino. Kantigas ebreas para ninyos traduzidas $i$ kantadas en ladino. Cantigas hebreas para niños traducidas y cantadas en Ladino, trad. en judéo-espagnol S. Refael, Ha-reshut ha-leumit le-tarbut ha-ladino/Autoridad Nasionala del Ladino i su Cultura, Jérusalem 2018.

\title{
THE STAKES OF LITERARY TRANSLATION IN PERIPHERAL AND POST-VERNACULAR LANGUAGES: THE CASE OF JUDEO-SPANISH
}

\begin{abstract}
In this contribution, we study a small corpus of contemporary literary translations into the Judeo-Spanish language, considered both peripheral and post-vernacular, in order to understand the directions and specificities of the literary import into this language, the roles played by these translations, and the motivations of the actors involved in the translation process.

We proceed in four steps: (1) first, we study the historical context of both Judeo-Spanish language and the literature created in it; (2) secondly, we present their diametrically different situation after the Second World War; (3) against this background, we present the identified translations and analyse their formal and editorial characteristics (authors, publishers, translators, original languages,
\end{abstract}


dates of publication, formats, layouts), as well as their paratexts; (4) finally, we study the results of this analysis in the light of both concepts of peripherality and post-vernacularity.

Key words: Judeo-Spanish language, literary translations, literary import, post-vernacularity, peripherality. 